\title{
Transient and Steady-State Shear Banding in a Lamellar Phase as Studied by Rheo-NMR
}

\author{
9 By Bruno Medronho ${ }^{1}$, Ulf Olsson², Claudia Schmidt ${ }^{3}$, and Petrik Galvosas ${ }^{4, *}$ \\ 1 Laboratory of Plant Biotechnology, Faculty of Sciences and Technology, University of Algarve, \\ Campus de Gambelas, Bd. 8, 8005-139 Faro, Portugal \\ 2 Physical Chemistry, Lund University, PO Box, 124, 22100 Lund, Sweden \\ 133 Department of Chemistry, Faculty of Science, University of Paderborn, Warburger Straße 100, 33098 \\ 14 Paderborn, Germany \\ $15{ }^{4}$ MacDiarmid Institute for Advanced Materials and Nanotechnology, School of Chemical and Physical \\ 16 Sciences, Victoria University of Wellington, PO Box, 600, 6140 Wellington, New Zealand
}

17 Dedicated to Professor Hans-Wolfgang Spiess on the occasion of his 70th birthday

(Received July 4, 2012; accepted in revised form September 21, 2012)

(Published online

21

\section{Shear-Induced Structure / Lamellar Phase / NMR Velocimetry / NMR Diffusometry / ${ }^{2} \mathrm{H}$ NMR Spectroscopy}

25

26 Flow fields and shear-induced structures in the lamellar $\left(\mathrm{L}_{\alpha}\right)$ phase of the system triethylene 27 glycol mono $n$-decyl ether $\left(\mathrm{C}_{10} \mathrm{E}_{3}\right)$ /water were investigated by NMR velocimetry, diffusometry, 28 and ${ }^{2} \mathrm{H}$ NMR spectroscopy. The transformation from multilamellar vesicles (MLVs) to aligned 29 planar lamellae is accompanied by a transient gradient shear banding. A high-shear-rate band of aligned lamellae forms next to the moving inner wall of the cylindrical Couette shear cell while 30 a low-shear-rate band of the initial MLV structure remains close to the outer stationary wall. The

31 band of layers grows at the expense of the band of MLVs until the transformation is completed.

32 This process scales with the applied strain. Wall slip is a characteristic of the MLV state, while 33 aligned layers show no deviation from Newtonian flow. The homogeneous nature of the opposite transformation from well aligned layers to MLVs via an intermediate structure resembling undulated multilamellar cylinders is confirmed. The strain dependence of this transformation appears to be 35 independent of temperature. The shear diagram, which represents the shear-induced structures as 36 a function of temperature and shear rate, contains a transition region between stable layers and 37 stable MLVs. The steady-state structures in the transition region show a continuous change from ${ }_{38}$ layer-like at high temperature to MLV-like at lower temperature. These structures are homogeneous on a length scale above a few micrometers.

\section{Introduction}

Applications of NMR methods in rheological investigations of complex fluids have become more and more popular over the last 20 years [1-5]. NMR spectroscopy can be

* Corresponding author. E-mail: petrik.galvosas@vuw.ac.nz 
1] used to probe shear-induced orientations and structures [6,7] while NMR velocimetry 22 yields the flow field in Newtonian and non-Newtonian fluids [8,9]. Further structural 3 information is provided by studying self diffusion and its anisotropy in samples with 4 shear-induced structures [10-16]. These rheo-NMR methods have the advantage that 5 no additional molecules or particles, which may affect the sample properties, are needed 6 as probes and that no requirements concerning the optical properties of the samples 7 exist.

8 For spectroscopic studies of anisotropic fluids such as thermotropic and lyotropic 9 liquid crystals deuterium NMR is most convenient. The spectra of this $I=1$ nucleus 10 show a moderate quadrupole splitting and, therefore, are easy to measure and analyze, [11 yielding information about molecular orientation and dynamics [17]. Both spectroscopy [12 on bulk samples and spatially resolved spectroscopy have been used in the field of 13 rheo-NMR. Spatial resolution (NMR imaging) may be achieved by means of slice 14 selection, phase or frequency encoding, or a combination of these methods [9]. The 15 NMR measurement of diffusion and flow is based on the magnetic-field dependence 16 of the resonance frequency and, similar to spectroscopic imaging, requires magnetic 17 field gradients. Most commonly, the technique of pulsed field-gradient NMR is used, 18 which enables signal detection in a homogeneous magnetic field, yielding full spec19 tral resolution [9]. In the pulsed-gradient spin-echo (PGSE) [18] and pulsed-gradient 201 stimulated-echo (PGSTE) [19] experiments a pair of pulsed field gradients leads to 21 a random phase shift and thus a reduced echo intensity (in the case of diffusion) and 22. a coherent phase shift (in the case of flow).

23. Complex fluids, such as polymers, surfactant solutions, and liquid crystals, are of 24 interest both from a fundamental and a technological point of view [20]. The coup25 ling between structure and flow gives rise to interesting phenomena which may alter 26 the flow properties of a sample considerably and are therefore of relevance in the pro277 cessing and design of complex fluid materials. Among the lyotropic liquid crystalline 28 phases the lamellar phase is perhaps the most interesting one. Under shear the stacks of 29 amphiphilic bilayers may align with their normal either parallel to the velocity gradi30 ent or parallel to the vorticity axis. These orientations have become known as the "c" 31 and "a" orientation, respectively [21]. In addition, a defect structure resembling a dense 32 packing of multilamellar vesicles (MLVs or onions) is found in many cases. Roux and [33 coworkers have shown that the size of these onions is controlled by the applied shear 34 rate [22-25], often decreasing as the inverse square root of the shear rate [23,26]. 35 A large number of studies on shear-induced multilamellar vesicles has followed ( $c f$. ref[36 erences in [16], [26], and [27]) but the processes of MLV formation and destruction 37 are still not well understood. Deuterium NMR spectroscopy using $\mathrm{D}_{2} \mathrm{O}$ as a probe was 38 found to be a convenient method to investigate both the shear-induced orientations of 39 the lamellar phase and the formation of multilamellar vesicles in situ [26-28]. The $\mathrm{D}_{2} \mathrm{O}$ 40 spectra of the "normal" lamellar phase shows a quadrupole splitting, which depends on 41 the orientation of the layers with respect to the magnetic field. When MLVs are formed, 42 the quadrupole doublet collapses to a broad single peak because the water self-diffusion 43 along the curved bilayers of the onion shells and the rotation of the water molecules (44) connected with it occurs just on the right time scale for partial motional narrowing of 45 the spectra. As the MLVs become smaller upon increasing shear rate the line width of 46 the NMR spectra decreases. Therefore, the MLV size can be estimated from the width 

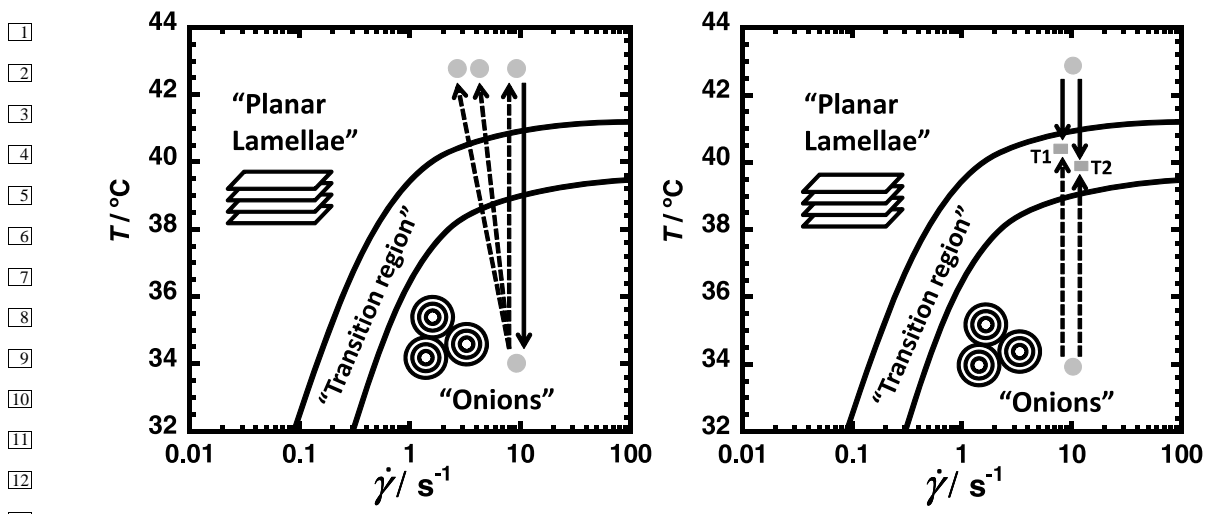

Fig. 1. Shear diagram of the system $\mathrm{C}_{10} \mathrm{E}_{3} / \mathrm{D}_{2} \mathrm{O}(40 \%$ w/w surfactant) [30] showing the stability regions of planar lamellae and MLVs (onions) and the initial and final states of the "jump" experiments. On the left, 15 the experiments involving jumps across the transition region are shown, whereas on the right the jumps into 16 the transition region are indicated.

17

18

(19) of the spectra [29]. The characteristic length scale of the MLV structure can also be 200 derived from NMR diffusometry [15].

21] One of the MLV-forming systems most studied to date is the binary solution of 22 the nonionic surfactant triethylene glycol mono $n$-decyl ether $\left(\mathrm{C}_{10} \mathrm{E}_{3}\right)$ in $\mathrm{D}_{2} \mathrm{O}$ contain23 ing 40 wt. \% surfactant [16,27,29-37,37-43], although shear-induced MLVs can be 24 found in all surfactants of the $\mathrm{C}_{n} \mathrm{E}_{m}$ type studied so far [26,28,33,44-55]. The shear 25 diagram of $\mathrm{C}_{10} \mathrm{E}_{3} /$ water ( $40 \mathrm{wt}$. \%), showing the orientation states as a function of tem26 perature and shear rate, is presented in Fig. 1 [30]. This system has shear regimes of 27 well aligned layers and MLVs in an easily accessible range of temperatures and shear 28 rates. All of our more recent NMR studies on MLVs have been carried out on this 29) system $[15,16,27,29,43]$.

30

${ }^{2} \mathrm{H}$ NMR spectroscopy under shear has revealed that, while the transformation of [31 aligned planar layers into MLVs is continuous and homogeneous, the reverse process 32 of MLV destruction occurs via the nucleation and growth of aligned layers [27]. Spa33 tially resolved ${ }^{2} \mathrm{H}$ NMR and diffusometry has shown that the layers nucleate at the inner 34 moving wall of a Couette cell [16]. By measuring the strain dependence of the diffusion [35 anisotropy in the shear-induced states [16] we could follow the pathway of MLV for36 mation and confirm that an intermediate structure resembling undulated multilamellar 37 cylinders aligned in the velocity direction occurs [32,33,56].

[38 Here, we report additional experiments on the $\mathrm{C}_{10} \mathrm{E}_{3}$ /water system, using NMR [3. velocimetry to study the dynamics of the system in addition to structural investiga40 tions using diffusometry and spectroscopy. By means of velocimetry the phenomenon 41] of shear banding [57-60] can be investigated. Gradient shear banding, that is the oc42 curence of bands with different shear rates, stacked along the velocity gradient, is 43 a likely scenario during the inhomogeneous MLV-to-layer transition [16] since MLVs 44 and aligned lamellae have different viscosities. There has also been a report on the ob(45) servation of vorticity shear banding, i.e., of bands of different stress stacked along the 46 vorticity axis, during the lamellar-to-onion transition [61] but we have seen no evidence 
1) of this in our NMR studies. In addition, we will present NMR experiments carried out to reveal the structure in the "transition region" which separates the two regimes of shearinduced aligned lamellae at high temperature and MLVs at low temperature, shown in Fig. 1.

\section{Materials and methods/experimental}

\subsection{The system $\mathrm{C}_{10} \mathrm{E}_{3} / \mathrm{D}_{2} \mathrm{O}$}

10 Triethylene glycol mono $n$-decyl ether $\left(\mathrm{C}_{10} \mathrm{E}_{3}\right)$ was purchased from Nikko Chemical 1 Co. (Tokyo, Japan) (purity higher than 99.8\%). Deuterium oxide $\left(\mathrm{D}_{2} \mathrm{O}\right)$ with a pu[12 rity of $99.9 \%$ was supplied by Sigma Chemicals (Steinheim, Germany). Samples were ${ }_{13}$ prepared by weighing $40 \mathrm{wt} \%$ of surfactant and $60 \mathrm{wt} \%$ of water into vials. The two 14 components were thoroughly mixed and subsequently centrifuged in order to remove $\left[15\right.$ air bubbles. All samples were prepared with a ratio $\mathrm{D}_{2} \mathrm{O} / \mathrm{H}_{2} \mathrm{O}$ of $9: 1$ by weight.

17

\section{2.2 NMR apparatus and rheo-NMR devices}

All experiments have been conducted on a Bruker Avance spectrometer operating at a ${ }^{1} \mathrm{H}$ resonance frequency of $400 \mathrm{MHz}$. Gradients of up to $1.45 \mathrm{~T} / \mathrm{m}$ for NMR diffusometry and velocimetry were provided by a Micro2.5 three-axes micro-imaging system (Bruker). The temperature of the sample was kept constant via the conventional Bruker temperature control. However, to avoid temperature gradients over the sample volume, the temperature of the gradient coolant was set to the same temperature as the target sample temperature. Sample temperatures were within $\pm 0.5 \mathrm{~K}$ of the desired temperature which was verified once prior to the experiments via a temperature sensor situated in a liquid filled NMR sample tube in place of the Couette cell. The same setup allowed to confirm that temperature equilibration after temperature jumps was completed after $40 \mathrm{~min}$.

The properties of the surfactant solution were studied in the gap of a concentric cylinder rheo-NMR Couette device (see Fig. 2 left) with an inner rotating tube (outer diameter $16 \mathrm{~mm}$ ) placed inside an outer stationary tube (inner diameter $18 \mathrm{~mm}$ ) resulting in a fluid gap of $1 \mathrm{~mm}$. Teflon spacers in the gap between cylinders ensure concentricity and smooth rotation of the inner cylinder. The outer, stationary tube is held by the NMR coil assembly while the inner tube is turned by a stepper motor via a gear box (with a ratio $50: 1$ ) and a rigid mechanical drive shaft [1]. The stepper motor is able to apply rotations in the frequency range $0.1 \mathrm{~Hz}$ to $14 \mathrm{~Hz}$ which generates shear rates of interest for the different experiments. Gap-average shear rates were calculated as

$$
\dot{\gamma}=\frac{r_{\mathrm{i}} \omega_{\mathrm{i}}}{r_{\mathrm{o}}-r_{\mathrm{i}}}
$$

(44 valid for small gap sizes [62]. The radii of the outer and inner cylinders are $r_{\mathrm{o}}$ and $r_{\mathrm{i}}$, 45 respectively, while $\omega_{\mathrm{i}}$ is the inner cylinder angular velocity. Actual shear rates deviate 46 by about $\pm 10 \%$ from the gap-average. 

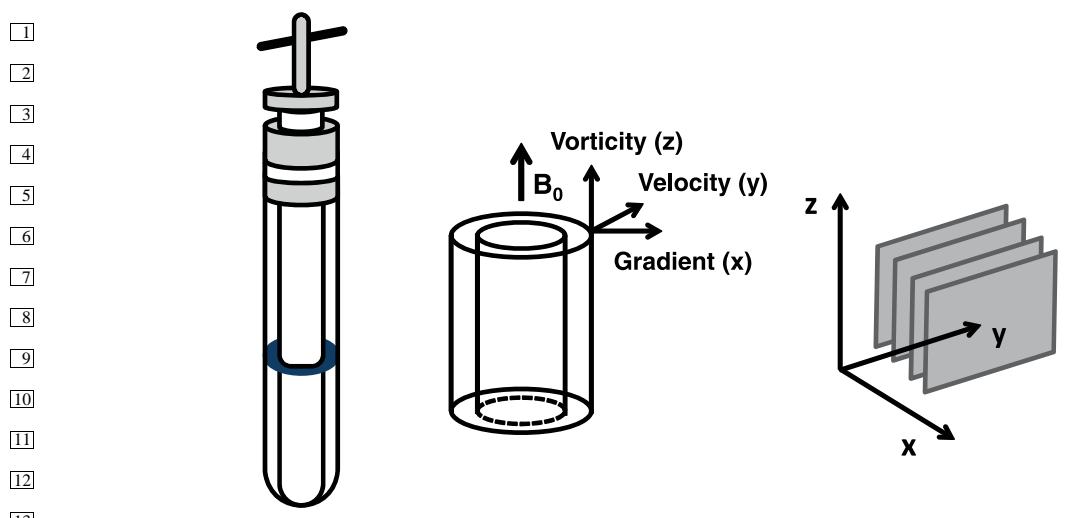

Fig. 2. Schematic illustration of the Couette geometry used (left). The schematic in the middle relates the shear frame with its velocity gradient, velocity and vorticity directions to $x, y$ and $z$ of the laboratory coordinate system defined by the static magnetic field $(z)$ and the magnetic field gradients $(x, y$, and $z)$. On

16 the right the orientation of aligned planar lamellae (in the "c" direction) in the shear frame is shown.

17

\subsection{Rheo-NMR experiments}

\subsubsection{Velocimetry}

1D NMR imaging was used in combination with the PGSTE for the acquisition of the velocity field across the Couette cell gap in the sheared sample [63]. The NMR pulse sequence used is shown in Fig. 3. The two gradients in the PGSTE part are incremented in steps simultaneously. This creates a residual phase shift after the second gradient pulse which is a measure of the velocity $v_{y}$ in the direction of the magnetic field gradient expressed by the collective displacement $\Delta y=v_{y} \Delta$ of the molecules during the observation time $\Delta$ :

30

$$
\Delta \phi=\gamma g_{y} \delta \Delta y
$$

3 Here, $g_{y}$ and $\delta$ are the gradient strength and width, respectively. $\gamma$ is the gyromagnetic 34 ratio. Actual velocities may be extracted from the measurement either by direct eval35 uation of Eq. (2) using recorded residual phase shifts $\Delta \phi$ or by using the concept of 36 dynamic NMR microscopy [64]. This concept utilises Fourier transforms with respect 37 to the imaging gradients as well as the PGSE gradients. The latter delivers the averaged 38 propagator and the mean velocity with it while the former provides spatial resolution 39 and is accomplished by the imaging part of the pulse sequence using a double slice se40 lection scheme. The first slice is positioned across the centre of the cell, oriented along [4] the $x, z$-plane with a width of $2 \mathrm{~mm}$. This selects two opposite strips from the annular 42 gap, ensuring that influences from the curvature of the Couette cell can be neglected, al43 lowing one to operate in a Cartesian coordinate system of velocity gradient $(x)$, velocity 44 $(y)$ and vorticity $(z)$ as indicated in Fig. 2 . The second slice is oriented perpendicular to 45 the first one along the $x, y$-plane with a width of $40 \mathrm{~mm}$, excluding undesired parts of 46 the sample such as the semi-spherical end of the Couette cell. 1D imaging is performed 


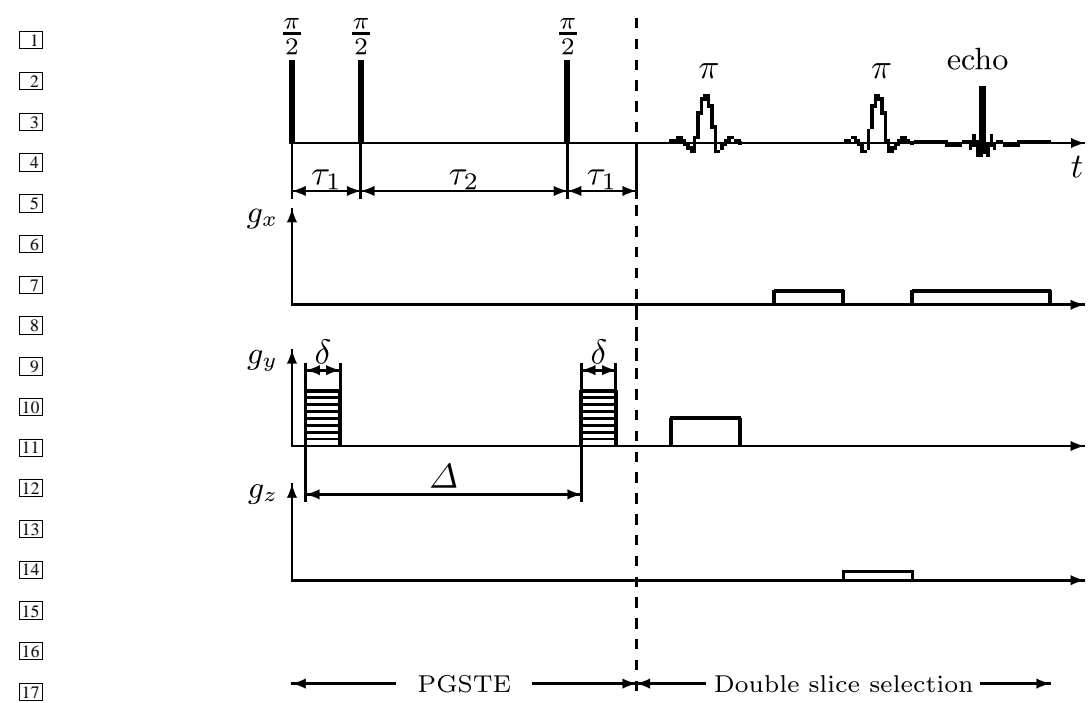

18 Fig. 3. Radiofrequency and magnetic field gradient pulse sequences for the acquisition of velocity fields. 19 Two magnetic field gradient pulses of duration $\delta$ and separation $\Delta$ are applied in the $y$-direction and increased successively in amplitude. Two soft $180^{\circ}$ pulses are used in combination with magnetic field slice gradients in the $y$ and $z$-direction to select a specific volume of spins.

22

233 via frequency encoding in $x$ direction along the velocity gradient, ultimately recording 24 and mapping the velocity field across the gap of the cell in the two strips.

25] Velocity images (maps) were recorded during transitions from the MLV to planar 26 lamellae at shear rates of $10 \mathrm{~s}^{-1}, 5 \mathrm{~s}^{-1}$ and $2.5 \mathrm{~s}^{-1}$. The opposite transition from lamellae 27] to MLVs was studied at $10 \mathrm{~s}^{-1}$. Likewise, for a shear rate of $10 \mathrm{~s}^{-1}$ velocity fields were 28 acquired after jumping into the transition region. Corresponding maps were obtained 29 with a spatial resolution of $100 \mu \mathrm{m} /$ pix resulting in a resolution of 10 pixels across the (30) gap. The observation time was set to $\Delta=20 \mathrm{~ms}$ and the gradient width to $\delta=1 \mathrm{~ms}$. 4 31. PGSE gradient values were used up to a maximum gradient of $g_{y}^{\max }=0.44 \mathrm{~T} / \mathrm{m}$ (for the 32 smallest shear rate). A total of 4 scans were acquired for each PGSE gradient step with 33 a repetition time of $6 \mathrm{~s}$, resulting in a total time of $96 \mathrm{~s}$ per velocity image. To follow the 34 velocity field in the gap over time images were recorded continuously (up to 80) while 35 the sample was under constant shear.

36 .

\section{2.3.2 Diffusometry}

38

39 The spatially resolved measurement of diffusivities (diffusion imaging) is very simi-

(40 lar to the concept of velocity imaging. In fact it uses the same pulse sequence (with 41 the only alteration that the PGSTE gradient pair in Fig. 3 may also be applied in $x$ or $42 z$ direction for measuring the corresponding diffusivities). The main difference is that (43) measurements are carried out while the sample is at rest. This results in a start-stop 444 experimental protocol with periods of shearing (increasing the strain) interspersed by 45 periods of zero shear while acquiring data. It was verified by previous studies that the (46) structure is quenched into a metastable state for at least $30 \mathrm{~min}$ [16], which allows for 
the time required by the diffusion experiments. This holds even for rapid transitions which are completed typically after several thousand strain units, corresponding to only several hundred seconds at a shear rate of $10 \mathrm{~s}^{-1}$.

$4 \quad$ It is also evident that no residual coherent phase shift as expressed by Eq. (2) will 5 occur. Instead diffusive motion will cause a random phase spread which relates to the 6 diffusion coefficient $D$ via the Stejskal-Tanner equation [18]

$$
E=E_{0} \exp \left\{-D(\gamma \delta g)^{2}\left(\Delta-\frac{1}{3} \delta\right)\right\}=E_{0} \mathrm{e}^{-D b} .
$$

$10 E_{0}$ is the signal amplitude without pulsed magnetic field gradients applied. It is con11 venient to introduce the so called $b$-factor, as given by Eq. (3), which represents all 12 parameters controlled by the experiment. The diffusion images were recorded with 13 similar parameters as compared to the velocity images. However, the gradient width and 14 amplitude of the PGSTE pair was increased to $\delta=5 \mathrm{~ms}$ and $g^{\max }=1.33 \mathrm{~T} / \mathrm{m}$ (16 gradi15 ent steps) which accounts for the smaller displacements caused by diffusion only. The 16 number of scans is increased from 4 to 8 .

17 As for the velocity images the obtained data sets are two-dimensional. The image 18 dimension is processed via Fourier transform leading to $1 \mathrm{D}$ images with 10 pixels 19 across the Couette cell gap (identical to the velocity images). The second dimension 20 (along the increasing PGSTE gradients), however, does not obey Eq. (2) anymore. In21. Stead this dimension was now processed by fitting Eq. (3) to the obtained data leading 22 to the diffusion coefficients for each individual pixel. The interested reader may find 23 further details on the experimental conditions and the data processing in [16]. Some of 24 the acquired data did not fit satisfactorily to a mono-exponential decay as expressed by

${ }^{25}$ Eq. (3). While this may indicate slight structural inhomogeneities across the sample it 26 was outside the scope of this study to investigate this behavior further. Therefore, the 27 averaged diffusion coefficient as obtained from the initial signal decay was used in these 28 cases.

29

\section{$2.4^{2} \mathrm{H}$ NMR}

32. The quadrupolar splitting of the deuterium nucleus was used to assess the amount of 33 order in the sample as characteristic for the different structures. Since planar lamel34 lae are globally oriented in the gap with their director being perpendicular to $z$ one 35 expects to see a doublet in the ${ }^{2} \mathrm{H}$ NMR spectra of heavy water. This doublet results 36 from the residual quadrupole coupling due to the anisotropic environment of the heavy 37 water molecules. Its splitting reflects both the orientation of the phase axis (here the 38 orientation of the layer normal, being perpendicular to the magnetic field) and the local 39 orientational order parameter of heavy water (which is about one tenth of the order pa40 rameter of the surfactant). The situation for MLVs is different since the lamellar layers [41 are strongly curved. Therefore all orientations of the surface director will be present 42 in the sample and diffusion of the water molecules along the surface results in partial 43 motional narrowing, leading to only a single peak in the deuterium spectra as a charac44 teristic signature for MLVs [28].

45 Deuterium spectra have been acquired with a spectral width of $5 \mathrm{kHz}$ allowing to 46 record the wide spectra originating from the MLVs. Spectra were acquired continuously 
11 while the sample was under shear, thus recording the structural change in the sample over time.

\section{Results and discussion}

\subsection{Shear banding during the transition from multilamellar vesicles to planar layers}

According to the shear diagram of $\mathrm{C}_{10} \mathrm{E}_{3} / \mathrm{D}_{2} \mathrm{O}$ (40 wt. \% surfactant) shown in Fig. 1, [10 MLVs are not stable at temperatures above about $41^{\circ} \mathrm{C}$. Thus, when a sample consist(11 ing of shear-induced MLVs is sheared at higher temperature the MLVs are destroyed [12 and a state of planar lamellae aligned parallel to the velocity-vorticity plane is obtained. 13 This shear-induced transition has previously been found to resemble a nucleation-and14 growth process, in which the layers start to grow from the inner moving wall of the 15 Couette cell [16]. Using spatially resolved NMR diffusometry and velocimetry the 16 MLV-to-layer transition is investigated here in more detail. The protocol for these ex17 periments is represented by the arrows shown in Fig. 1, left. As indicated by the arrows 18 pointing upwards, MLVs were initially prepared by shearing at $\dot{\gamma}=10 \mathrm{~s}^{-1}$ and $34^{\circ} \mathrm{C}$ for (19) $60 \mathrm{~min}$. Then shear was stopped, the temperature was changed to $43^{\circ} \mathrm{C}$, where MLVs 200 are not stable under shear, and after 45 min to allow for temperature equilibration, shear [2] was started again at the desired shear rate. While the sample continued to be sheared 222 when measuring velocities, shear was interrupted for diffusion measurements, employ23 ing the start-stop protocol described in Sect. 2.3.2 above.

24 Figures 4 to 7 show the results obtained when the formation of layers takes place 25 at $\dot{\gamma}=10 \mathrm{~s}^{-1}$ (vertical upward arrow in Fig. 1, left). As reported previously [16], spa26 tial inhomogeneities during the transformation process lead to diffusivities that vary [27 as a function of the radial position across the annular gap of the shear cell. This can 28 be also seen in Fig. 4, in which profiles of the diffusivity in the velocity direction are 29 presented for selected strain values. In this and all the following viewgraphs depicting [30 spatial profiles, the wall of the inner moving cylinder is to the left at $0 \mathrm{~mm}$ and the outer [31 stationary wall is positioned at $1 \mathrm{~mm}$. The diffusion profiles reveal that the formation of [32 layers, having a higher diffusivity along $y$ than MLVs, does not occur simultaneously 33 everywhere in the gap. Already at the lowest strain of 200, a region with the higher 34 diffusion coefficient corresponding to layers is observed close to the inner wall. As $\gamma$ in55 creases, the interface between the regions of layers and MLVs moves towards the outer [36 stationary wall of the Couette cell, until the transition is completed and $D$ is the same [37 everywhere in the gap. This is in good agreement with our previous diffusion results 38 obtained with another shear cell, possessing a somewhat wider gap of $1.5 \mathrm{~mm}$, and by [39 ${ }^{2} \mathrm{H}$ NMR imaging [16]. At $\gamma=1800$ a second narrow region of fast diffusion can be ob40 served at the outer wall, $c f$. Fig. 4. Similar observations, typically at higher strains, were (41) made in additional diffusion experiments not presented here, but not by velocimetry. We 42 will return to this issue later.

433 Since aligned layers have a lower viscosity than MLVs $[25,26]$ one can expect 44 the shear rate to be higher in the part of the sample consisting of layers. By means (45) of velocity imaging we have investigated whether the observed regions with different 46 structures do indeed correspond to bands of different shear rate. In a separate experi- 


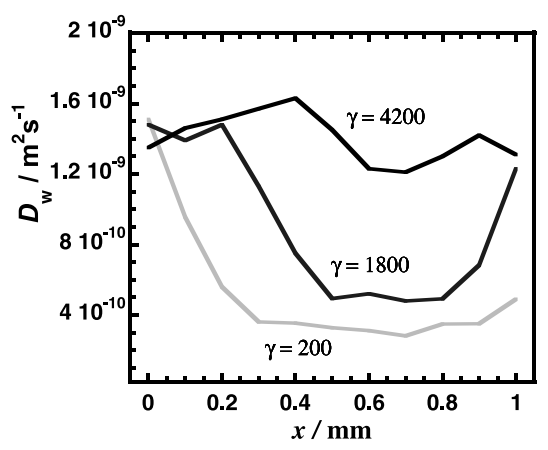

Fig. 4. Diffusion coefficient along the velocity direction as a function of the position in the gap for different strain values during the transition from MLVs to planar lamellae at $43{ }^{\circ} \mathrm{C}$ and $\dot{\gamma}=10 \mathrm{~s}^{-1}$.

14

15

16

17

18

19

20.

21

22.

23

24

25

26.

27

28

29

30.

31

32

33

34

35

36.

37.

38

39.

40

41

42

43

44.

45

46 rates are obtained by linear fits.
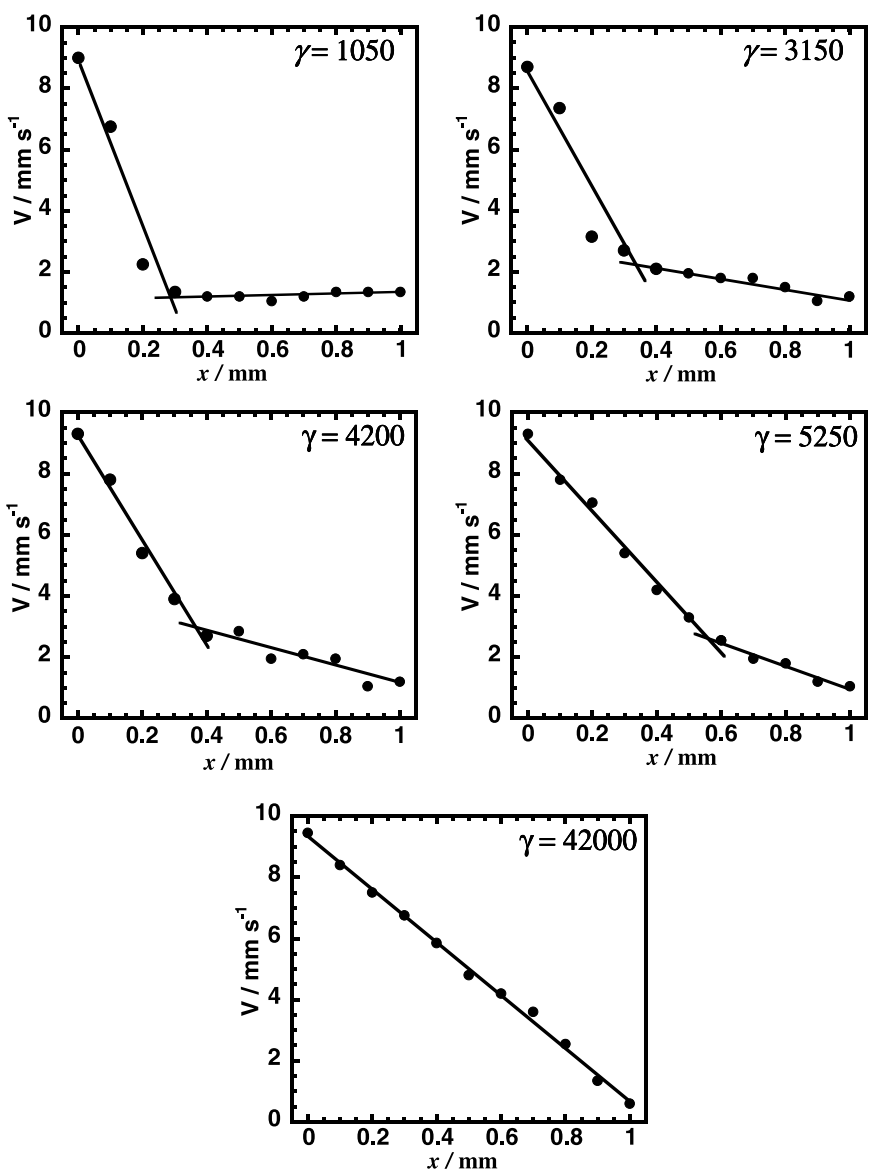

Fig. 5. Velocity profiles across the gap for different strain values during the transition from MLVs to planar amellae at $43^{\circ} \mathrm{C}$ and $\dot{\gamma}=10 \mathrm{~s}^{-1}$. Bands of low and high shear rate can be distinguished. The average shear 47 
Fig. 6. Average shear rates in the two bands during the transition from MLVs to planar lamellae at $43^{\circ} \mathrm{C}$ and $\dot{\gamma}=10 \mathrm{~s}^{-1}$.

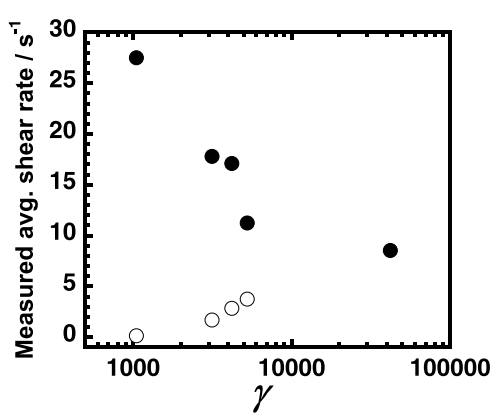

13

14

15

16

17

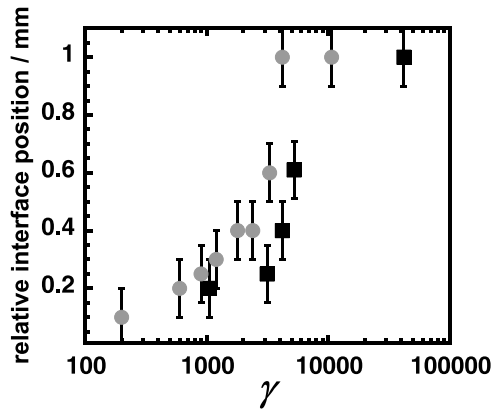

25 Fig. 7. Position of the interface between planar lamellae and MLVs obtained by diffusometry (gray circles) 26 and between the bands of high shear rate and low shear rate determined by velocimetry (black squares) 27] during the transition from MLVs to planar lamellae at $43{ }^{\circ} \mathrm{C}$ and $\dot{\gamma}=10 \mathrm{~s}^{-1}$.

28

29.

30 ment, starting again from MLVs generated at $34{ }^{\circ} \mathrm{C}$ by shearing at $\dot{\gamma}=10 \mathrm{~s}^{-1}$, the [31 profiles of the velocity component in the direction of flow were measured. The results 32 are shown in Fig. 5. At lower strains, two regions of different slopes, indicating two 33 bands of different shear rates, can be clearly distinguished. The interface between the 34 band of high shear rate moves from the inner wall towards the outer wall until, at large 35 strain, there is only one region of uniform shear rate. The average shear rates in each (36 band, obtained by linear regression as shown in Fig. 5, are presented in Fig. 6.

In Fig. 7, the interface between the two shear bands extracted from the velocity pro8 files in Fig. 5 is compared with the interface between the regions of different diffusion 39 coefficients as obtained by diffusometry ( $c f$. Fig. 4). The results from the two different 40 methods agree very well. In our previous work a fairly good agreement between the in[41 terface positions observed by diffusometry and ${ }^{2} \mathrm{H}$ NMR imaging was found [16]. We 42 can thus conclude that, within experimental error, the shear rate bands correspond to 43 bands of different structures.

44 Velocimetry monitoring the MLV-to-layer transition starting from the same initial (45) state as before was also performed at shear rates of 5 and $2.5 \mathrm{~s}^{-1}$ ( $c f$. additional upward 46 arrows in Fig. 1, left). The results are summarized in Fig. 8. The velocity profiles shown 

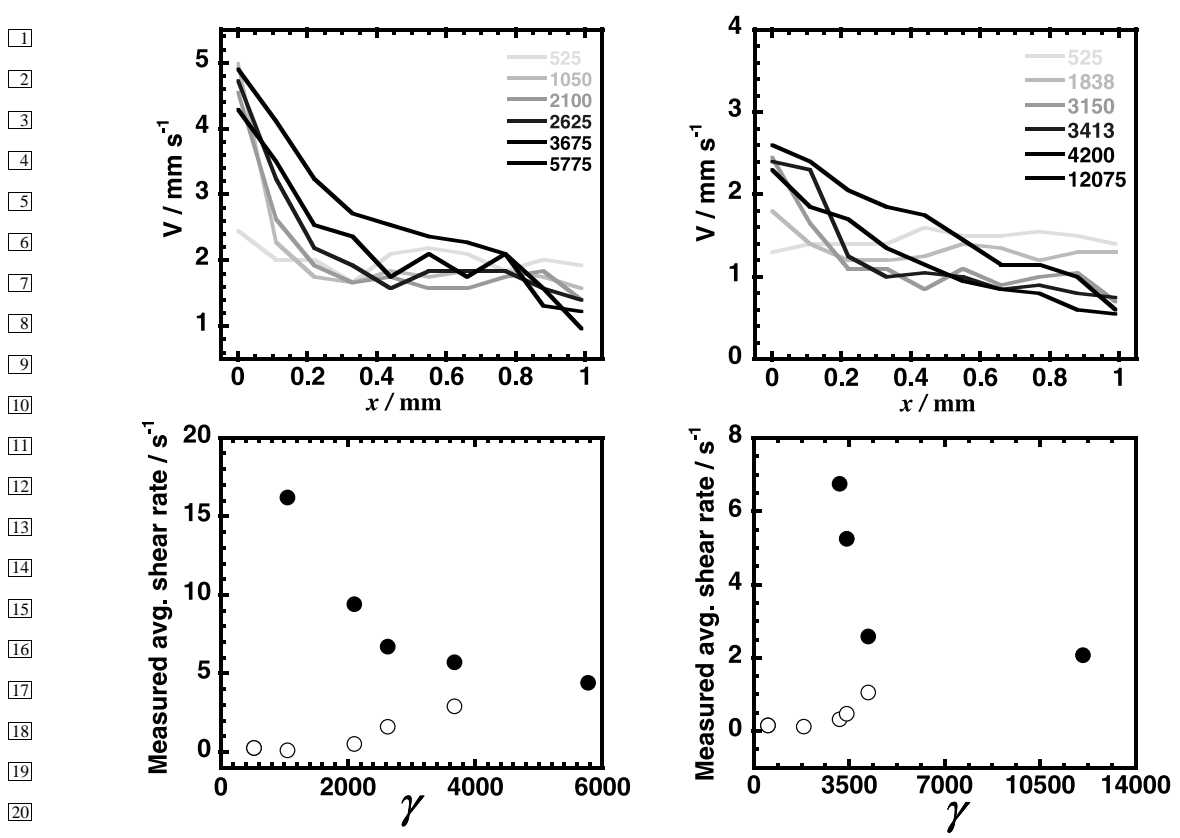

Fig. 8. Velocity profiles (top) and average shear rates (bottom) during the transition from MLVs to planar lamellae at $43^{\circ} \mathrm{C}$ and $\dot{\gamma}=5 \mathrm{~s}^{-1}$ (left) and $2.5 \mathrm{~s}^{-1}$ (right).

23

24

25. on top of Fig. 8 illustrate once more that the band of low shear rate at the outer wall,

26 which is initially present and corresponds to MLVs, shrinks with increasing strain. This

27 shrinkage matches the creation and growth of a high-shear-rate band near the wall of

28 the rotating cylinder, until a single band corresponding to planar layers is obtained. The

29 shear rates extracted by linear fitting of the velocity profiles are presented in the bottom

30 of Fig. 8. The results are in keeping with the observations at the higher shear rate.

31 The boundaries between the shear bands obtained for all three shear rates inves32. tigated by velocimetry are compared in Fig. 9. When the position of the boundary is 33 plotted as a function of strain the data fall on one curve, demonstrating that the MLV-to34 layer transition scales with the applied strain and does not depend on the applied shear 35 rate. This is in agreement with previous findings for the same system based on an an[36 alysis of ${ }^{2} \mathrm{H}$ NMR line shapes [27]. Strain scaling has also been observed in the case of 37 shear-induced alignment of the lyotropic hexagonal phase $[65,66]$.

38

\section{3.2 Shear effect on the defect density}

411 In the previous section we have discussed the shear-induced destruction of vesicles and

42 their transformation into planar lamellae. Before turning to the reverse transformation

43 from layers into vesicles, we will stay for a moment within in the stability regime of

44 layers and consider the influence of shear on the structure of the system. Previously,

45 shear flow had been observed to induce structural defects in the lamellar structure even

46 if no subsequent transition to MLVs follows [43] and the shear-rate was determined as 


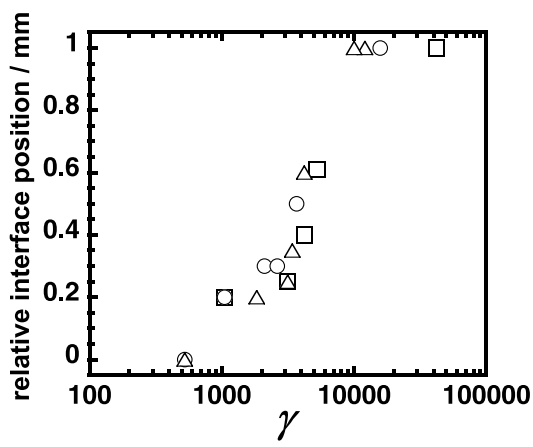

Fig. 9. Position of the interface between the bands of low and high shear rate during the transition from MLVs to planar lamellae at $43^{\circ} \mathrm{C}$ for the three different shear rates investigated. Triangles, circles, and squares correspond to shear rates of $2.5,5$, and $10 \mathrm{~s}^{-1}$, respectively.

15

16

Fig. 10. Profiles of the water diffusion coefficients in the velocity (left) and vorticity (right) direction as a function of the applied shear rate at $43^{\circ} \mathrm{C}$ within the region of planar lamellae after shearing at rates of $2810,0.1$, and again at $10 \mathrm{~s}^{-1}$. The black, grey, and dotted lines correspond to measurements after the first 29 period of shear at $10 \mathrm{~s}^{-1}$, after shear at $1 \mathrm{~s}^{-1}$, and after the second period of shear at $10 \mathrm{~s}^{-1}$, respectively.

[32 the key parameter to control the defect density. Both ${ }^{2} \mathrm{H}$ NMR and rheology data sug[33 gested a reversible increase of the defect density as the shear rate was increased (within (34 the lamellar region). Here, we investigate if these previously observed pretransitional (35) defects are large enough to have an influence on the diffusion behavior. In one of the ex36 periments described in the previous section, after the MLV-to-lamellae transition being [37 completed at a shear rate of $10 \mathrm{~s}^{-1}$, the shear rate was decreased to $0.1 \mathrm{~s}^{-1}$ and, after38 wards, increased back to $10 \mathrm{~s}^{-1}$. Diffusion was measured after each shearing period, that [39 is, at $10,0.1$, and $10 \mathrm{~s}^{-1}$. Diffusion maps for the velocity and vorticity directions are 40 shown on the left and right of Fig. 10, respectively. The diffusion profile for the velocity (41) direction shows no perceptible changes. In the vorticity direction, however, diffusion 42 becomes faster, when the shear rate is decreased. This change is reversible. Comparison 43 of the $D_{\mathrm{w}}$ values for the different spatial directions shows that they are equal at the low 44 shear rate. This is in good agreement with the model of nearly perfectly planar lamellae. 45. The slower diffusion along the vorticity axis at the higher shear rate, on the other hand, 46 points to structural defects which have a preferred orientation. A structure consistent 


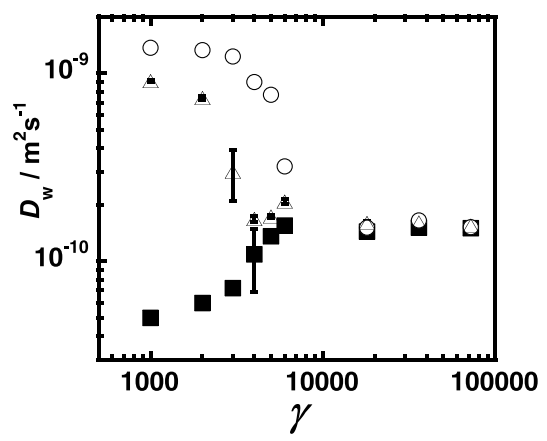

Fig. 11. Diffusion coefficients of water along the velocity (circles), vorticity (triangles), and velocity gradient (squares) directions during the transition from planar lamellae to MLVs at $34^{\circ} \mathrm{C}$ and $\dot{\gamma}=10 \mathrm{~s}^{-1}$.

14

15 with the observed diffusion anisotropy are undulations with a wave vector in the vor16 ticity direction. Such undulated structures have been suggested to occur also during the 17 transformation form planar lamellae to MLVs at higher shear rates in the MLV regime 18 of the shear diagram [16]. Thus, the pretransitional behavior observed in the regime of (19 planar layers shows steady-state structures which are very similar to the transient inter20 mediate structures postulated for the early stage of the lamellae-to-onion transition at 21] higher shear rates.

\subsection{Homogeneous transition from planar layers to multilamellar vesicles}

25. We now turn to the complete transformation from planar layers to vesicles in the MLV 26 region of the shear diagram as indicated by the arrow pointing downwards in the left 27 panel of Fig. 1. The initial state was prepared by shearing at $43^{\circ} \mathrm{C}$ and a shear rate of $2810 \mathrm{~s}^{-1}$ for $60 \mathrm{~min}$. Then shear was stopped, the temperature was changed to $34^{\circ} \mathrm{C}$, and 29 after thermal equilibration shear was started again at $10 \mathrm{~s}^{-1}$. The diffusometry results 30 are summarized in Figs. 11 and 12.

[1] Figure 11 shows the strain dependence of the coefficients for water diffusion in the [32 velocity, velocity gradient, and vorticity directions $\left(D_{\text {vel }}, D_{\text {grad }}\right.$, and $D_{\text {vort }}$ represented 33 by circles, squares, and triangles, respectively). Besides a global increase of the dif34 fusion constants, which is expected because of the higher temperature, Fig. 11 shows 35 the same features of the strain-dependence of the diffusion anisotropy as reported pre36 viously for a temperature of $25^{\circ} \mathrm{C}$ [16]. Thus, these new data are consistent with the 37 previously described model of structural transformation: The initially planar lamellae 38 $\left(D_{\text {vel }}=D_{\text {vort }}>D_{\text {grad }}\right)$ at first become undulated, with the wave vector along the vor[99 ticity axis $\left(D_{\text {vel }}>D_{\text {vort }}>D_{\text {grad }}\right)$. Subsequently, undulations with wave vectors along 40 the velocity directions are added. The fission and fusion of the bilayer membranes oc411 curs in an anisotropic way such that cylinder-like structures $\left(D_{\text {vel }}>D_{\text {vort }}=D_{\text {grad }}\right)$ are 42 formed, which finally transform into the isotropic $\operatorname{MLV}$ structure $\left(D_{\text {vel }}=D_{\text {vort }}=D_{\text {grad }}\right)$. 433 Even the strain scale, on which these processes occur, is the same for both tempera44 tures. Figure 12 illustrates the change of the diffusion profiles across the gap during 45 the transformation process (for the diffusion in the velocity direction, which under46 goes the largest change). Throughout the transformation, $D_{\mathrm{w}}$ remains independent of 


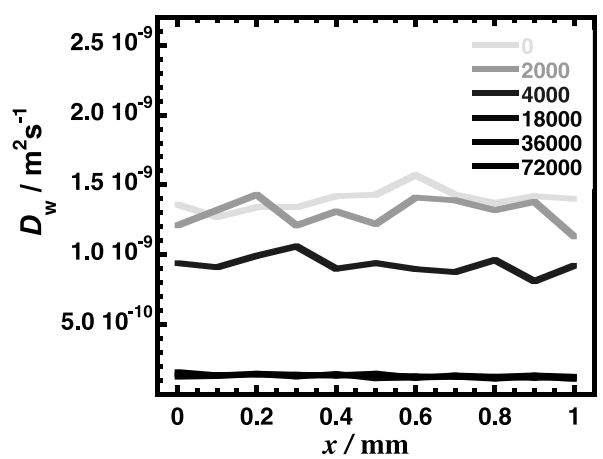

Fig. 12. Diffusion coefficient of water along the velocity direction as a function of the position in the gap 13 for different strain values during the transition from planar lamellae to MLVs at $34{ }^{\circ} \mathrm{C}$ and $\dot{\gamma}=10 \mathrm{~s}^{-1}$.

14

15

16

17
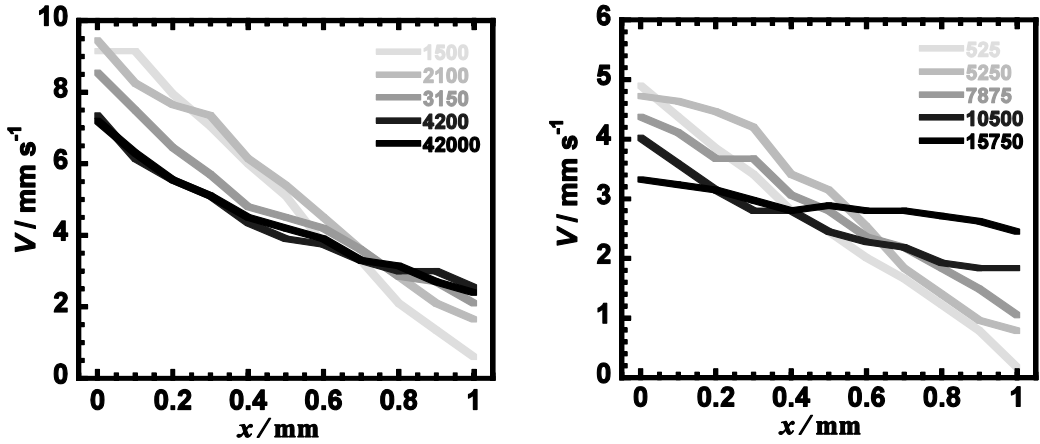

Fig. 13. Velocity profiles during the transition from planar lamellae to MLVs at $34^{\circ} \mathrm{C}$ and shear rates of $2810 \mathrm{~s}^{-1}$ (left) and $5 \mathrm{~s}^{-1}$ (right).

29

30

[3] the position, confirming that the transformation from planar layers to vesicles is a ho32 mogeneous process.

[33 In Fig. 13 velocity profiles are presented for the transition from planar layers to 34 vesicles. The profiles on the left were obtained at the same shear rate $\dot{\gamma}=10 \mathrm{~s}^{-1}$ as the (35) diffusion data, while the profiles on the right were obtained in another experiment, car(36 ried out at $\dot{\gamma}=5 \mathrm{~s}^{-1}$. As the transformation evolves, a gradual decrease in the slope 37 of the velocity profile (corresponding to a decrease of the actual shear rate) combined 38 with increasing wall slip is observed. With increasing strain, a curvature in the velocity (39 profiles is noticed, in particular, at the higher shear rate, but there is no indication of 40 discrete shear bands.

41

42

\subsection{Structures in the transition region}

4 4 The so called transition region depicted in the shear diagram of Fig. 1 is based on meas45 urements of the steady-state viscosities as a function of shear rate [30]. It represents the 46 region of shear rates, where shear thickening is observed, opposed to the shear thinning 
प] of lamellae becoming aligned and onions becoming smaller in size [26]. Rheo-SANS 22 experiments indicate that planar lamellae and onions may coexist here [33]. In most 33 later investigations this region, which seems to separate the regimes of planar lamellae 4 and MLVs, was deliberately avoided $[16,27,29,43]$. In the following we turn our atten5 tion towards this transition region. The structures at two points in this region, labeled $6 \mathrm{~T} 1$ and T2 (shown in the right part of Fig. 1) are investigated. Diffusion coefficients, ${ }^{2} \mathrm{H}$ 77 NMR spectra of heavy water, and velocities are analyzed. In total, four different types 8 of jump experiments have been performed, such that each of the points T1 and T2 is 9 approached starting from the stability regime of either planar lamellae (at higher tem10 perature) or MLVs (at lower temperature). Thus we can clarify whether the steady-state (111 structures in the transition region depend on the initial conditions.

[12 All experiments were performed at a shear rate of $10 \mathrm{~s}^{-1}$. The initial states of MLVs $\left[13\right.$ and planar lamellae were prepared at 34 and $43{ }^{\circ} \mathrm{C}$, respectively, by shearing for one 114 hour. The temperatures of the target points are $40^{\circ} \mathrm{C}$ for $\mathrm{T} 1$, which is close to the (15) regime of stable layers, and $39^{\circ} \mathrm{C}$ for $\mathrm{T} 2$ in the middle of the transition region. As in 16 the experiments discussed before, the samples were sheared at the initial temperature, 17 then shear was stopped during the temperature "jump" and the samples were allowed 18 to thermally equilibrate for at least $40 \mathrm{~min}$ before, finally, shear was started again and 19 the structural transformation at the target temperature was followed as a function of [20) strain.

21] None of the four experiments revealed a discontinuity in the diffusion profiles 22 across the gap indicating that the transformation process is homogeneous in all cases.

23 Thus, if there is a coexistence of planar layers and vesicles, both structures are homoge24 neously distributed in the Couette gap. This is clearly different from the case described 25 in Sect. 3.1 above, in which the jump is made from a state of MLVs into the stability 26 region of planar lamellae, $c f$. Fig. 4.

27 Figure 14 shows how the anisotropy of the diffusion coefficients (averaged over the 28 gap) evolves as a function of strain. The upper and lower rows of plots display the data 29 for the target points $\mathrm{T} 1$ and $\mathrm{T} 2$, respectively, while the left and right columns repre30 sent the different initial conditions, that is, MLVs and aligned layers, respectively. Note [31 that for the right column strain increases from right to left so that the diffusivities at the 32 respective target points are shown "face to face" in the middle of each row.

33 When starting from MLVs and jumping to T1 (upper left of Fig. 14, diffusion ini34 tially is nearly isotropic as expected for MLVs but it becomes anisotropic, with a small 35 increase of $D_{\mathrm{w}}$ in both velocity and vorticity directions and a simultaneous decrease 36 in the gradient direction. Hence, the data seem to indicate a progressive formation of 37 a lamellar-like structure. However, the process is much slower and the final diffusion 38 anisotropy is smaller compared with a jump across the transition region. When T1 is [30 approached from an initial state of aligned lamellae (upper right of Fig. 14) the initial 40 anisotropy is reduced somewhat. A slight decrease in $D_{\mathrm{w}}$ in the velocity and vorticity 41 directions is accompanied by an increase in $D_{\mathrm{w}}$ in the velocity gradient direction. Also, 42 the difference between the diffusivities in the velocity and vorticity directions becomes 43 larger. The structural changes when approaching T1 from aligned layers appear to be 4 much less pronounced compared to the MLV-to-T1 transition. However, for all three 45 spatial directions, the final diffusion coefficients are the same, independent of the initial 46 structure of the system. 

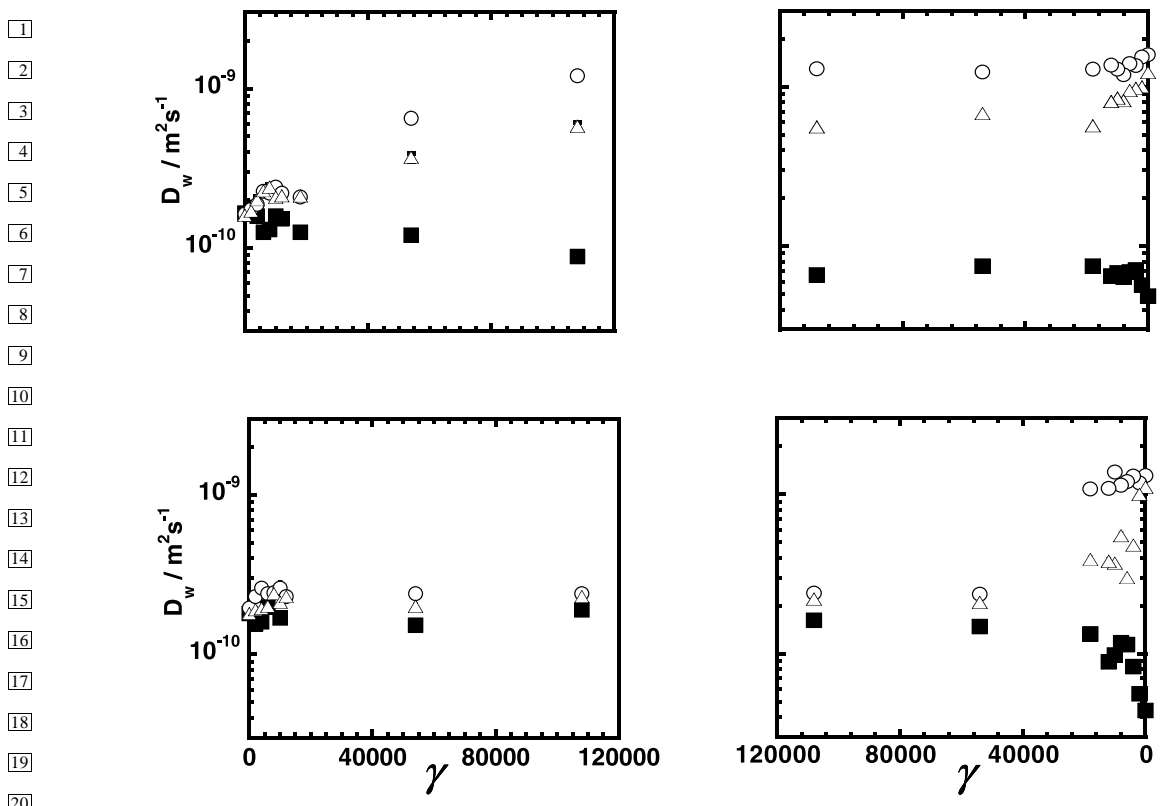

Fig. 14. Diffusion coefficients of water during the transitions to points T1 (top) and T2 (bottom) in the transition region of the shear diagram, starting from different initial states. Left: the initial state consists of MLVs generated at $34{ }^{\circ} \mathrm{C}$, right: initial state of planar lamellae generated at $43{ }^{\circ} \mathrm{C}$. The strain axis has 3 been reversed in the right column for better comparison of corresponding final states at high strain. The cir24 cles, triangles, and squares represent diffusion coefficients in the velocity, vorticity, and velocity gradient 25. direction, respectively.

26

27]

28

This is also the case for T2 as target point. After shearing, an almost isotropic struc(30) ture is obtained. When the target state is approached starting from MLVs there is hardly 31 any change in the $D_{\mathrm{w}}$ values, except for a slight overall increase in the beginning of the 32 transformation at strains below 1000. When starting from layers and jumping to T2 the (33) initial anisotropy vanishes almost completely. Altogether, the diffusivities suggest that 34 the structure of $\mathrm{T} 1$ is closer to layers, while T2 resembles MLVs. This indicates a grad35 ual change of the steady-state structures as temperature is changed within the transition 36 region.

In Fig. 15, the ${ }^{2} \mathrm{H}$ NMR spectra of heavy water in the initial and final states of the 38 jump experiments are compared. The left and right parts of Fig. 15 show the experi39 ments with T1 and T2, respectively, as final state. In each case, solid and dotted lines 40 (with higher maximum) correspond to transformations starting from aligned layers and (41) MLVs, respectively. Thus, they represent the initial states - a broad single peak for 42 MLVs and a doublet for planar lamellae. The two superimposed spectra of lower height 43 in each set were obtained when the samples had reached their final states. The small (44) shoulder in the left peak arises most likely from a poorly mixed sample which leads to 45 the superposition of slightly different spectra. This is often observed and does not affect 46 onion formation. 

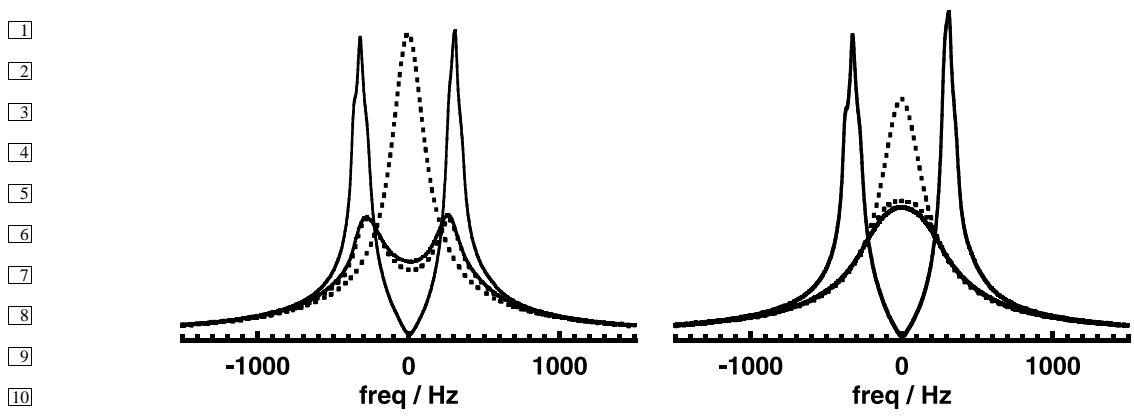

Fig. 15. Initial and final ${ }^{2} \mathrm{H}$ NMR spectra for the transformations observed at points $\mathrm{T} 1$ (left) and T2 (right)

12 in the transition region. In each case, solid lines represent the transformation starting from aligned lamellae

13 (doublet with large splitting) and dotted lines correspond to the transformations starting from MLVs (sin-

14 gle peak with higher maximum intensity). The spectra of the final states have an intermediate shape that is

15 different for $\mathrm{T} 1$ and $\mathrm{T} 2$ but independent of the initial structure.

16

The almost perfect agreement of the superimposed spectra makes evident that the 19 final state does not depend on the history of the samples. For both T1 and T2 as target 20 points, the final structure is distinctly different from both initial structures. While the 21 spectra of T1 still show a splitting, the spectra of T2 consist of a single but very broad 22 peak. In other words, the steady-state structure at the higher temperature (T1) is more 23 similar to planar lamellae than to MLVs, whereas the one at the lower temperature (T2) 24 resembles MLVs, in good agreement with the diffusometry results.

25. Although the spectra of the final states T1 and T2 can be fitted by superpositions of 26 three Lorentzian lines (corresponding to one doublet and a broad central peak) they are 27 clearly not a sum of the spectra observed in the two regions above and below the tran28 sition region. The spectral splitting of the doublet in state $\mathrm{T} 1$ is much smaller than the 29 splitting in the regime of aligned lamellae and suggests a less ordered state. Likewise, 30 the spectra in state T2 are much broader than the MLV spectra at lower temperatures, 31 indicating that the bilayers comprising the structure are less curved than in the MLV 32 region. There is also no evidence of shoulders which would indicate an underlying 33 doublet resulting from planar lamellae. Therefore, we can rule out the coexistence of 34 large domains of aligned layers and MLVs in the transition region. The NMR spec35 tra point towards a homogeneous structure, intermediate between perfect layers and 36 MLVs and changing continuously as one passes through the transition region. A su37 perposition of two structures appears less likely, although inhomogeneities on a length 38 scale smaller than a few $\mu \mathrm{m}$, as estimated from the mean square displacement of wa39 ter due to self diffusion during the time scale of the NMR measurement, cannot be 40 excluded.

41 The velocity profiles, depicted in Fig. 16 for the four experiments involving the 42 transition region, further support the model of well-defined steady-state structures in 43 the transition region. There is no evidence of shear banding. In the initial stage of the 44 transformation "MLV to T1" (Fig. 16, upper left), there is considerable wall slip, and 45 the shear rate decreases somewhat as the strain is increased. At a later stage, the shear 46 rate increases approximately to its applied value and the wall slip disappears. The first 

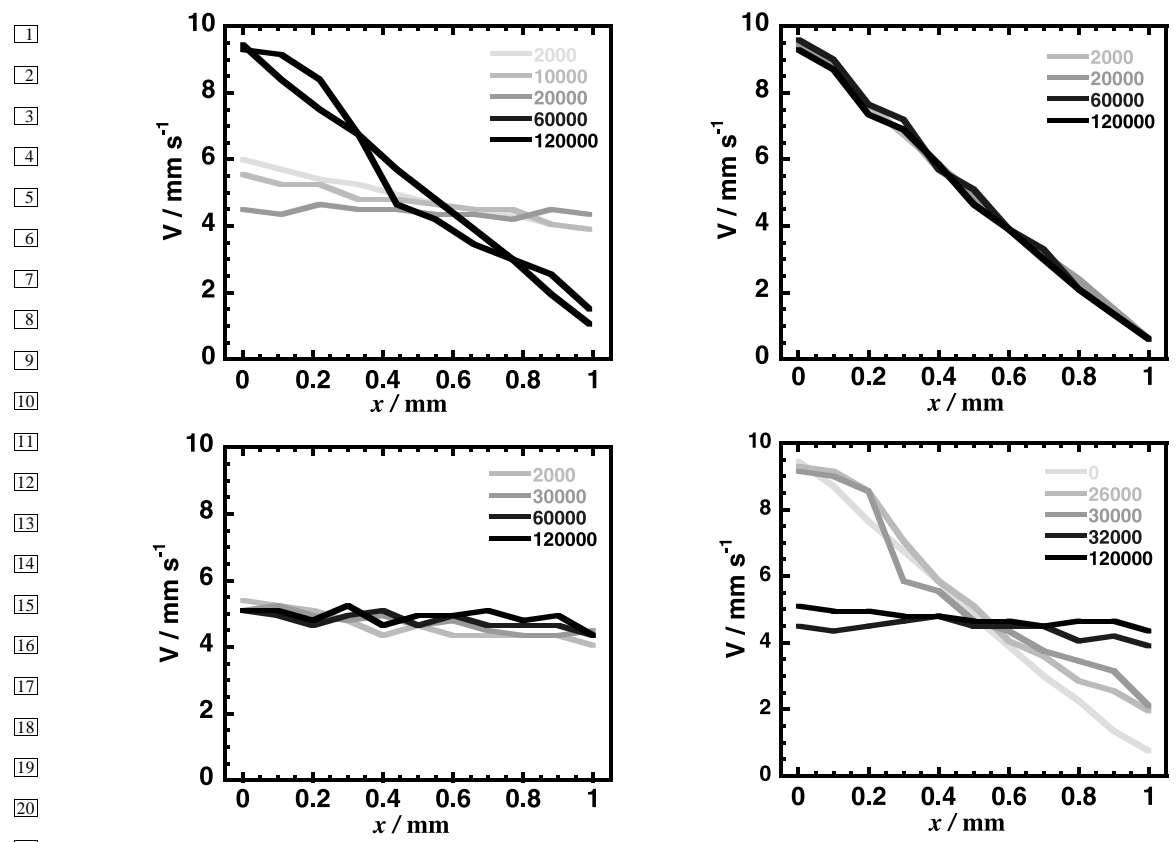

Fig. 16. Velocity profiles during the transitions to points $\mathrm{T} 1$ (top) and $\mathrm{T} 2$ (bottom) in the transition region of the shear diagram, starting from different initial states. Left: the initial state consists of MLVs generated 23 at $34{ }^{\circ} \mathrm{C}$, right: initial state of planar lamellae generated at $43{ }^{\circ} \mathrm{C}$

26 part of the process may indicate a growth of MLVs, e.g., by fusion of smaller vesicles. 27 Larger MLVs are known to exhibit a higher viscosity, hence the observed decrease of 28 the shear rate. Eventually, some of the MLVs may be disrupted and a matrix of aligned 29 planar lamellae may form. Due to the low viscosity of aligned lamellae the shear rate 30 increases.

[1] For the transition from planar lamellae to T1 (Fig. 16, upper right), no changes [32 in the velocity profile are observed and the curves for all strains fall on one line. 33 This is in agreement with the relatively small changes observed by diffusometry and 34 spectroscopy: the initial and final states are structurally related; also the final state is [35 layer-like.

(36) The velocity profiles obtained during the MLV-to-T2 transformation (Fig. 16, lower [37 left) confirm that there are no major changes during this transition. There is a very 38 small decrease of the average shear rate, which is much smaller than the applied shear (39 rate because of considerable wall slip. The decrease in shear-rate indicates an increase 40 in viscosity, which can be explained by the increase in MLV size as indicated by the 411 spectral line shapes.

42] Finally, the transformation from planar layers to T2 (Fig. 16, lower right) shows 433 a change from a high shear rate to lower shear rates with significant wall slip, just in 4 4 reverse to the changes observed during the MLV-to-T1 transformation. This is in agree45 ment with $\mathrm{T} 2$ being more closely related to MLVs than to planar layers, in agreement 46 with the results from diffusometry and spectroscopy. 


\subsection{Wall slip}

In many of the velocity profiles discussed here, wall slip is evident. In the absence of slip, the velocity at the stationary outer wall is zero and, for shear rates of 10,5 , and $2.5 \mathrm{~s}^{-1}$ and a gap of $1 \mathrm{~mm}$ thickness, it is 10,5 , and $2.5 \mathrm{~mm} \mathrm{~s}^{-1}$, respectively, at the inner moving wall. This is approximately given when the shear bands disappear during the MLV-to-layer transformation, seen in Figs. 5 and 8, and in all cases when the structure consists of planar lamellae, for instance, in the initial stages of the layer-to-MLV transformation (Fig. 13). On the other hand, MLVs always show strong wall slip, which is evident, for example, in Figs. 13 and 16. Although the velocity profiles may become fairly flat in the onion state, a small shear rate is always maintained within the sample.

III plug flow occurred the measured tangential velocity would slightly increase with the radius; this phenomenon is not observed. From an inspection of all velocity profiles we may conclude that wall slip is a characteristic feature of the MLV structure and does not occur in samples consisting of aligned layers.

We may now return to Fig. 4 and the increased diffusivity at the outer wall that is observed during the shear-banded transformation from MLVs to planar lamellae. If the velocity at the stationary outer wall is larger than zero and a contact between wall and sample remains there must be a third shear band with a very high shear rate close to the outer wall. This band obviously consists of aligned layers having high diffusivities. In some cases it is so narrow that it is missed in the experiments which have a limited resolution. Applying the so called lever rule, by which the total shear rate is calculated as a weighted sum over the observed bands, to the shear banding profiles during the MLVto-layer transition, $c f$. Fig. 5, we obtain total shear rates smaller than the applied values. 247 This provides further evidence of a missing narrow high-shear band at the outer wall.

In the MLV state slip occurs at both walls, reducing the measured velocity in the voxel at the inner wall and increasing it at the outer wall. Obviously, the major part of the total shear occurs close to the walls in layers too thin to be seen in our NMR velocimetry experiments, while the bulk of the sample undergoes very little shear.

30.

\section{Conclusions}

33 The rheo-NMR experiments reported here have shown that the transformation from 34 MLVs to aligned planar lamellae after a jump in temperature across the socalled transi35 tion region of the shear diagram is accompanied by a transient gradient shear banding. 36 Two bands can be observed: a band of high shear rate consisting of aligned lamellae 37 next to the moving wall of the shear cell and a band of low shear rate consisting of 38 the initial MLV structure close to the outer stationary wall. This shear banding scales [9. with the applied strain. Upon increasing strain, the boundary between the two bands is 40 shifted towards the outer wall until the MLV band disappears. High diffusivity and wall 411 slip at the outer wall as well as failure of the lever rule indicate the existence of an addi42 tional narrow band of high shear rate consisting of aligned layers at the outer wall. Wall 43 slip is found to be a characteristic and reproducible feature of the onion state.

44 No discrete shear bands are resolved by NMR velocimetry when MLVs are formed 45 during the homogeneous transformation starting from an initial state of well aligned 46 layers. However, a formation of regions of high shear rate at both walls must be pos- 
tulated to account for the observed wall slip and the low actual shear rate evident from the velocity profiles in the MLV state.

The shear-induced structures are independent of the history of the samples. This holds true also in the transition region. Here, steady states are found, the structures of which change continuously from being layer-like at higher temperatures to onion-like at lower temperatures. NMR spectroscopy suggests that the structures in the transition region are homogeneous on a length scale above a few micrometers.

\section{Acknowledgement}

This work was supported by grants from the Swedish Research Council and the Royal Society of New Zealand (Marsden grant). BM thanks Fundação para a Ciência e tecnologia (FCT) for a post-doc grant (ref. SFRH/BPD/74540/2010).

\section{References}

1. P. T. Callaghan, Rep. Prog. Phys. 62 (1999) 599.

2. C. Schmidt, Is , in: Modern Magnetic Resonance, G. A. Webb (Ed.) Springer, New York (2008), pp. 1515-1521.

3. P. T. Callaghan, Curr. Opin. Colloid. In. 11 (2006) 13.

4. N. Dingenouts and M. Wilhelm, Korea-Australia Rheol. J. 22 (2010) 317.

5. H. S. Melito and C. R. Daubert, Ann. Rev. Food Sci. Technol. 2 (2011) 153.

6. A. I. Nakatani, M. D. Poliks, and E. T. Samulski, Macromolecules 23 (1990) 2686.

7. D. A. Grabowski and C. Schmidt, Macromolecules 27 (1994) 2632.

8. Y. Xia and P. T. Callaghan, Macromolecules 24 (1991) 4777.

9. P. T. Callaghan, Principles of Nuclear Magnetic Resonance Microscopy, Clarendon Press, Oxford (1993).

10. A. Lutti and P. T. Callaghan, Phys. Rev. E 73 (2006) 011710.

211. A. Lutti and P. T. Callaghan, J. Magn. Reson. 180 (2006) 83.

33 12. A. Lutti and P. T. Callaghan, J. Magn. Reson. 187 (2007) 251.

34 13. A. Lutti and P. T. Callaghan, Eur. Phys. J. E 24 (2007) 129.

35 14. A. Lutti and P. T. Callaghan, Appl. Magn. Reson. 33 (2008) 293.

56 15. I. Åslund, B. Medronho, D. Topgaard, O. Söderman, and C. Schmidt, J. Magn. Reson. 209 (2011) 291.

38 16. B. Medronho, J. Brown, M. G. Miguel, C. Schmidt, U. Olsson, and P. Galvosas, Soft Matter 7 39 (2011) 4938.

40 17. H. W. Spiess, Adv. Polym. Sci. 66 (1985) 23.

41 18. E. O. Stejskal and J. E. Tanner, J. Chem. Phys. 42 (1965) 288.

42 19. J. E. Tanner, J. Chem. Phys. 52 (1970) 2523.

43 20. R. G. Larson, The Structure and Rheology of Complex Fluids, Oxford University Press, New 44 York, Oxford (1999).

45 21. C. R. Safinya, E. B. Sirota, R. F. Bruinsma, C. Jeppesen, R. J. Plano, and L. J. Wenzel, Science 261 (1993) 588.

47 22. O. Diat and D. Roux, J. Phys. II 3 (1993) 9.

48 23. O. Diat, D. Roux, and F. Nallet, J. Phys. II 3 (1993) 1427.

49 24. O. Diat, D. Roux, and F. Nallet, J. Phys. IV 3(C8) (1993) 193.

50 25. D. Roux, F. Nallet, and O. Diat, Europhys. Lett. 24 (1993) 53.

51 26. S. Müller, C. Börschig, W. Gronski, C. Schmidt, and D. Roux, Langmuir 15 (1999) 7558.

522 27. B. Medronho, S. Shafaei, R. Szopko, M. G. Miguel, U. Olsson, and C. Schmidt, Langmuir 24 (2008) 6480.

54 28. M. Lukaschek, S. Müller, A. Hasenhindl, D. A. Grabowski, and C. Schmidt, Colloid Polym. Sci. 274 (1996) 1. 
29. B. Medronho, C. Schmidt, U. Olsson, and M. G. Miguel, Langmuir 26 (2010) 1477.

30. C. Oliviero, L. Coppola, R. Gianferri, I. Nicotera, and U. Olsson, Colloid. Surface. A 228 (2003) 85.

31. T. D. Le, U. Olsson, K. Mortensen, J. Zipfel, and W. Richtering, Langmuir 17 (2001) 999.

32. J. Zipfel, F. Nettesheim, P. Lindner, T. D. Le, U. Olsson, and W. Richtering, Europhys. Lett. 53 (2001) 335.

33. F. Nettesheim, J. Zipfel, U. Olsson, F. Renth, P. Lindner, and W. Richtering, Langmuir 19 (2003) 3603.

34. F. Nettesheim, U. Olsson, P. Lindner, W. Richtering, J. Phys. Chem. B 108 (2004) 6328.

35. B. Medronho, S. Fujii, W. Richtering, M. G. Miguel, U. Olsson, Colloid Polym. Sci. 28 (2005) 317.

36. S. Fujii and W. Richtering, Eur. Phys. J. E 19 (2006) 139.

11 37. B. Medronho, M. G. Miguel, and U. Olsson, Langmuir 23 (2007) 5270.

38. S. Koschorek, S. Fujii, and W. Richtering, Prog. Theor. Phys. Suppl. 175 (2008) 154.

39. S. Koschoreck, S. Fujii, P. Lindner, and W. Richtering, Rheol. Acta 48 (2009) 231.

40. S. Fujii, S. Koschorek, P. Lindner, and W. Richtering, Langmuir 25 (2009) 5476.

14 41. S. Fujii, Prog. Colloid Polym. Sci. 136 (2009) 69.

15 42. L. Filippelli, B. Medronho, C. O. Rossi, M. G. Miguel, and U. Olsson, Mol. Cryst. Liq. Cryst. $16 \quad 500$ (2009) 166.

177 43. M. Medronho, B. Rodrigues, M. G. Miguel, U. Olsson, and C. Schmidt, Langmuir 26 (2010) 11304.

44. R. Weigel, J. Läuger, W. Richtering, and P. Lindner, J. Phys. II 6 (1996) 529.

45. T. D. Le, U. Olsson, and K. Mortensen, Phys. Chem. Chem. Phys. 3 (2001) 1310.

20 46. T. Kato, K. Miyazaki, Y. Kawabata, S. Komura, M. Fujii, and M. Imai, J. Phys.: Condens. 21] Matter 17 (2005) S2923.

[22 47. K. Miyazaki, Y. Kosaka, Y. Kawabata, S. Komura, and T. Kato, J. Appl. Crystallogr. 40 (2007) s332.

23 48. Y. Suganuma, M. Imai, T. Kato, U. Olsson, and T. Takahashi, Langmuir 26 (2010) 7988.

24 49. Y. Kosaka, M. Ito, Y. Kawabata, and T. Kato, Langmuir 26 (2010) 3835.

25 50. L. Gentile, C. O. Rossi, U. Olsson, and G. A. Ranieri, Langmuir 27 (2011) 2088.

26 51. L. Gentile, K. Mortensen, C. O. Rossi, U. Olsson, and G. A. Ranieri, J. Colloid Interface Sci. 362 (2011) 1.

52. M. Ito, Y. Kosaka, Y. Kawabata, and T. Kato, Langmuir 27 (2011) 7400.

53. J. Zhou, J. Yang, X. Chen, W. Luo, W. Zhang, K. Yang, and B. Zhang, J. Mol. Struct. 987 (2011) 91.

30 54. L. Gentile, C. O. Rossi, and U. Olsson, J. Colloid Interface Sci. 367 (2012) 537.

31 55. L. Gentile, B. F. B. Silva, S. Balog, K. Mortensen, and U. Olsson, J. Coll. Interface Sci. 372 (2012) 32 .

56. G. Auernhammer and H. R. Brand, Phys. Rev. E 66 (2002) 061707.

57. P. D. Olmsted, Rheol. Acta 47 (2008) 283.

58. S. M. Fielding, Soft Matter 3 (2007) 1262.

59. S. Manneville, Rheol. Acta 47 (2008) 301.

36 60. P. T. Callaghan, Rheol. Acta 47 (2008) 243.

37 61. G. M. H. Wilkins and P. D. Olmsted, Eur. Phys. J. E 21 (2006) 133.

62. H. A. Barnes, J. F. Hutton, and K. Walters, An Introduction to Rheology, Elsevier, Amsterdam (1989).

63. S. A. Rogers and P. T. Callaghan, Rheol. Acta 48 (2009) 735.

40 64. P. T. Callaghan, C. D. Eccles, and Y. Xia, J. Phys. E 21 (1988) 820.

411 65. M. Lukaschek, D. A. Grabowski, and C. Schmidt, Langmuir 11 (1995) 3590.

[42 66. S. Müller, P. Fischer, and C. Schmidt, J. Phys. II 7 (1997) 421. 